\title{
L'EFFETTO DI PARETE NELLE CAMERE \\ DI IONIZZAZIONE
}

\author{
Pio Vitrozzi
}

Una delle più discusse correzioni da applicarsi nelle misure di radioattività mediante le camere di ionizzazione, è il così detto " effetto di parete ». Esso consiste nel fatto che una particella $\alpha$ emessa ad una distanza dalla parete della camera inferiore al " range », non può utilizzare tutto il suo percorso agli effetti della ionizzazione, entro un determinato angolo solido, dipendente dalla forma geometrica della parete e dalla distanza da essa a cui è stata emessa.

Per camere cilindriche non piccole fu proposta ( $\left.{ }^{1}\right)$ da Duane e Laborde $\left({ }^{2}\right)$, nel caso di presenza nella camera di sola emanazione di Ra, ossia all'istante iniziale della misura, la seguente formula empirica :

$$
J=K\left(1-0,517 \frac{0}{V}\right)
$$

in cui: $J$ è la radioattività misurata;

$K$ è il valore effettivo della radioattività;

$O$ è la superficie della camera di ionizzazione;

$V$ è il volume della camera.

Il Deij $\left(^{3}\right)$ affrontò la trattazione teorica dell'argomento, ma, date le enormi difficoltà analitiche, si accontentò di trattare il caso di una parete piana estendendo $i$ risultati al caso di parete cilindrica, il che può farsi in buona approssimazione, solo quando il raggio della superficie cilindrica è sufficientemente grande nei confronti del range.

Egli pervenne alla formula:

$$
\begin{gathered}
J=\lambda n K\left\{1-\frac{1}{2} \cdot \frac{R}{\rho} \cdot \frac{1}{K} \int_{0}^{\mathrm{R}} \frac{r}{R} \Phi(r) d r+\right. \\
\left.+\frac{1}{6} \cdot \frac{R^{\circ}}{\rho^{2}} \cdot \frac{1}{K} \int_{0}^{\mathrm{R}} \frac{r^{2}}{R^{2}} \Phi(r) d r\right\}
\end{gathered}
$$

nella quale: 
$\lambda$ è la costante di disintegrazione radioattiva della sostanza considerata presente nella camera;

$n$ è il numero di atomi radioattivi per $\mathbf{c m}^{3}$;

$K=\int_{0}^{\mathbf{R}} \Phi(r) d r$

$R$ è il " range " di una particella $\alpha$ nell'aria;

$\rho$ è il raggio del cilindro;

$\Phi(r)$ è il numero di coppie di ioni che viene generato lungo il percorso ad una distanza $r$ dal punto di emissione della particella.

Com'è noto la $\Phi(r)$, chiamata pure curva di ionizzazione o curva di Bragg, è stata dedotta sperimentalmente da diversi Autori, tra i quali G. II. Henderson $\left({ }^{4}\right)$ e I. Curie \& F. Behaunek $\left(^{5}\right)$ e pertanto, non essendo nota l'espressione analitica di essa, il Deij eseguiva graficamente gli integrali che figurano nella [2].

Essendo questo lo stato delle ricerche ho dedotto la formula teorica esatta per il calcolo dell'effetto di parete sia nel caso piano che nel caso cilindrico. Ho sviluppato inoltre il procedimento di calcolo nel caso di una piccola camera di ionizzazione, alla quale quindi non sarebbero applicabili le due suaccennate formule [1] e [2]. Precisamente ho preso in considerazione la camera di ionizzazione annessa all'apparecchio dello Schmidt (raggio $\mathrm{cm} \mathrm{4;} \mathrm{altezza} \mathrm{cm} \mathrm{6,5),} \mathrm{col} \mathrm{quale}$ il Prof. Imbò ha eseguito numerose ricerche di radioattività delle varie sorgenti dell'Isola d'Ischia $\left({ }^{6}\right),\left({ }^{7}\right),\left({ }^{8}\right)$ e $\left({ }^{9}\right)$.

Si è presa innanzi tutto in considerazione la $\Phi(r)$ allo scopo di tentarne una rappresentazione analitica. Si è scelta la curva sperimentale ottenuta da I. Curie per il RaC'. Com'è noto infatti, per ottenere quella relativa al Rn, si può far uso della parte finale della curva RaC'. Non è stato agevole rappresentare la $\Phi(r)$ analiticamente con una funzione unica in tutto il range; si sono dovuti perciò considerare quattro distinti intervalli, nei quali la funzione è stata rappresentata con sufficiente fedeltà rispettivamente da uma parabola quadratica, da una esponenziale, da una somma di esponenziali, da una retta.

Omettendo i complessi sviluppi analitici, si è pervenuti alle seguenti formule per il calcolo dell'effetto di parete.

Nel caso di parete cilindrica:

$$
I_{\mathrm{m}}=I\left\{1-\frac{N}{2 \pi R^{2} A} \int_{\mathbf{K}-\mathbf{a}}^{\mathbf{R}} \int_{0}^{\vartheta_{0}^{*}} \int_{0}^{\phi^{*}} \int_{r^{*}}^{\mathbf{a}} \sigma \cdot \cos \vartheta \cdot \Phi(r) \cdot d r \cdot d \sigma \cdot d \vartheta \cdot d \varphi\right\}
$$


in cui :

$\Phi(r)$ conserva il significato già precisato sopra;

$I_{\mathrm{m}}=$ radioattività misurata all'istante iniziale;

$I=$ radioattività effettiva;

$N=$ numero totale di coppie di ioni generate da una particella $\alpha$ che esaurisce il suo percorso nell'aria;

$\boldsymbol{R}=$ raggio del cilindro;

$$
A=\int_{0}^{\mathrm{a}} \Phi(r) d r
$$

$a=$ range della particella $\alpha$ nell'aria;

$$
\vartheta^{*}=\arccos \frac{R-\sigma}{a}
$$

$\sigma=$ distanza del punto di emissione della particella $\alpha$ dall'asse del cilindro;

$$
\varphi^{*}=\arccos \frac{R^{2}-\sigma^{2}-a^{2} \cos ^{2} \vartheta}{2 \sigma a \cos \vartheta}
$$

Nel caso di parete piana:

$$
I_{\mathrm{m}}=I\left\{1-\frac{N}{2 A h} \int_{0}^{\mathrm{a}} \int_{\tau}^{\mathrm{a}} \frac{\tau \psi(r)}{r^{2}} d r \cdot d \tau\right\}
$$

in cui, oltre $\mathrm{i}$ simboli già noti nel caso cilindrico, $\tau=$ distanza del punto di emissione della particella $\alpha$ dal piano;

$$
\psi(r)=\int_{\mathrm{r}}^{\mathrm{a}} \Phi(r) d r
$$

$h=$ distanza massima dal piano alla quale si estende l'emissione delle particelle $\alpha$.

Si sono poi confrontate le due formule già note con quelle dedotte e si sono avuti, per la camera di ionizzazione annessa allo Schmidt, di cui è cenno più sopra $(R=\mathrm{cm} 4$, altezza $1=\mathrm{cm} 6,5)$, i seguenti risultati:

A) - In base alle formule teoriche dedotte:

$$
I=-1,63 I_{\mathrm{m}} ;
$$


$B$ ) - In base alla formula empirica del Duane, valida per camere non troppo piccols:

$$
I=1,7: I_{\mathrm{m}}
$$

C) - In base alla formula del Deij che assimila la parete cilindrica a parete piana e quindi valida per camere di dimensioni sufficientemente grandi nei confronti del range:

$$
J=1,26 \quad I_{\mathrm{m}} \text {. }
$$

É ovvio che il metodo accennato è suscettibile di sviluppo ed estensione. A parte infatti la laboriosità dei calcoli, si potrebbe determinare l'andamento della correzione per l'effetto di parete, al variare del rapporto $\frac{l}{k}$ o anche, come nella formula del Duane, al variare del rapporto $\frac{O}{V}$ e una volta ottenuto il detto andamento, col metodo dei minimi quadrati, determinare l'espressione analitica della funzione che meglio rispecchi il detto andamento.

Si perverrebbe in tal modo ad una formula che consentirebbe di dedurre l'effetto di parete, note le dimensioni della camera di ionizzazione, analoga a quella del Duane, ma dedotta teoricamente e valida per camere di piccole dimensioni.

Napoli - Istituto di Fisica Terrestre dell'Università - Novembre 1955.

\section{RIASSUNTO}

Vengono dedotte le formule teoriche per il calcolo dell'effetto di parete nelle camere di ionizzazione per la misura della radioattività, prendendo in considerazione sia il caso della parete piana che il caso della parete cilindrica.

Si applicano le formule dedotte al calcolo dell'effetto di parete nella camera di ionizzazione annessa all'apparecchio dello ScHMIDT, alla quale, essendo di piccole dimensioni nei confronti del range della particella $\alpha$, non sono applicabili le formule già note del DUANE (empirica) e del DEIJ (approssimata), e si confrontano $i$ risultati che si ottengono con le tre formule.

Si accenna all'estensione del metodo applicato allo scopo di pervenire ad una relazione analitica che consenta di dedurre l'effetto di parete, note le dimensioni della ramera. 


\section{$S U M M A R Y$}

The theoretical formulae for the wall effect in the ionization chambers for radioactivity measurements are deduced, taking into consideration both the case of a flat wall and of a cylindrical wall.

These formulae are applied to the calculation of the wall effect in the ionisation chamber connected to the Schmidt apparatus; the known formulae by Duane (empirical) and by Deij (approximated) are in fact not applicable in this case, since the dimensions of the chamber are small compared to the $\alpha$ particle range.

The results obtained by the application of the three formulae are compared.

Mention is made of the extension of the method for establishing an analytical relation which allows to deduce the wall effect when the dimensions of the chamber are known.

\section{BIBLIOGRAFIA}

(1) Israel H., Radioaltivität. I, Johann Abrosius Bart, Verlag, Leipzig, 1940, pag. 65 .

(2) Duane W. \& Laborde A., C. R. 150, 1421, 1910; Journ. de Phys. 4, 605, 1905.

(3) DeiJ L. J. L., Metingen van het radongehalte in de atmosfeer. Tesi di laurea Università di Amsterdam, A. W. Sijthoff's Uitgeversmastschappij N. V., Leiden, 1939.

(4) Henderson G. H., The Range and Ionization of the $\alpha$ Partic'es from Radium $C$ and Thorium C. Philos. Mag., Vol. XLII S. VL 1921, pag. 538.

(5) Cunie I. \& Brnounex F, Etude de la conrbe de Bragg relative aux rayons du Radium C. Le Jouın. de Phys. et le Radium, S. VI, T. VII, 1926, pag. 125.

(0) Імвò G., Saggio radiometrico in Lacco Ameno (Isola d'Ischia). La ric. Scientif. A. X, n. 10, 1939, pag. 936.

(ד) Імво̀ G., Misure della radioattività di a'cune acque dell'Isola d'Ischia. Ibidem, A. X, n. 6, 1939, pag. 546.

(8) Імво̀ G., Ulteriori misure di radioattività delle acque di Lacco Ameno e di Cetaro. Ibidem, A. X, n. 11, 1939.

(9) Iмво̀ G, Ricerche geofisiche alle, Grotte di Pertosa. Rend. Acc. Sc. Fis. e Mat., Napoli, S. IV, Vol. X, 1939-40.

(10) Rossi B. B. \& Staun H. H., Ionization chambers and counters. McGrawHill book Company, Inc., New York, 1949. 